\title{
A ORIENTAÇÃO FARMACÊUTICA AO CONSUMO INDISCRIMINADO DO PARACETAMOL
}

\author{
Bianka Cárita Ferreira de Oliveira ${ }^{1}$ \\ Leonardo Guimarães de Andrade²
}

RESUMO: De acordo com a Agência Nacional de Vigilância Sanitária, o paracetamol deve ser usado com cautela, "sempre observando a dose máxima diária e o intervalo entre doses, conforme as recomendações contidas na bula, para cada faixa etária”. Sendo a farmácia uma instituição de saúde, de acesso fácil e gratuito, onde o usuário, muitas vezes, procura, em primeiro lugar, facilitando o aumento no consumo desses medicamentos e consequentemente o aumento do número de intoxicações por fármacos. Neste sentido o profissional farmacêutico tem o papel de orientar com o intuito de combater o a automedicação, deve estar preparado para atuar na atenção farmacêutica como estratégia para diminuir o uso desnecessário de medicamentos e, dessa forma, melhorar a adesão farmacoterapêutica.

Palavras-chave: Paracetamol. Automedicação. Farmacêutico.

ABSTRACT: According to the National Health Surveillance Agency, paracetamol should be used with caution, "always observing the maximum daily dose and the interval between doses, according to the recommendations contained in the package insert, for each age group". As the pharmacy is a health institution, with easy and free access, where the user often seeks, in the first place, facilitating the increase in the consumption of these drugs and, consequently, the increase in the number of drug intoxications. In this sense, the pharmacist has the role of guiding in order to combat self-medication, he must be prepared to act in pharmaceutical care as a strategy to reduce the unnecessary use of medications and, thus, improve pharmacotherapeutic adherence.

Keywords: Paracetamol. Self-medication. Pharmaceutical.

\section{INTRODUÇÃO}

Os medicamentos de venda livre são conhecidos também como Medicamentos Isentos de Prescrição (MIP) e são os de primeira escolha pela população para

\footnotetext{
Aluna do curso Bacharel em Farmácia da UNIG - Universidade de Nova Iguaçu. E-mail: biia.carita@gmail.com

${ }^{2}$ Enfermeiro/Odontologia pela UNIG - Universidade de Nova Iguaçu
} 
enfermidades (PINTO, et al., 2015). O paracetamol é um medicamento de venda livre, portanto, não é necessária receita médica para compra. Por ser de fácil acesso à população, e juntamente com a falta de conhecimento, sua automedicação se tornou um grande problema de saúde pública, sendo este, o responsável por inúmeros casos de intoxicação por doses elevadas, podendo causar inicialmente náuseas, sudorese, icterícia e vômitos. Segundo Araújo, et al., (2013), por se tratar de um medicamento não opioide, não causa dependência.

Figura r. Fórmula estrutural do paracetamol

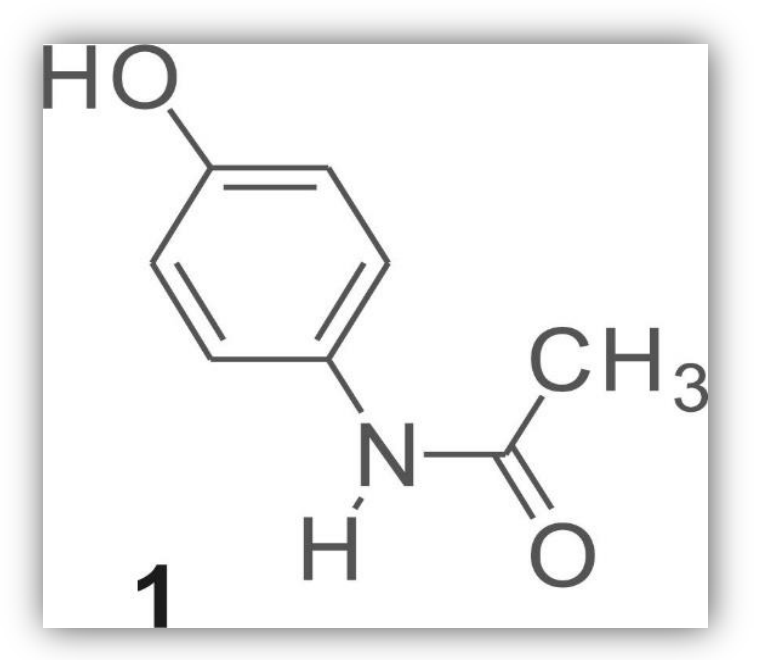

Fonte: Google Imagens.

O paracetamol possui propriedades analgésicas e antitérmicas, sendo utilizado em larga escala para o controle da dor. Sua ação fundamenta-se em inibir a síntese de substâncias endógenas, as cicloxigenases (COX-I e COX-2), enzimas capazes de induzir a atividade de prostaglandinas, o que culminaria em sintomas de inflamação. Além disso, há um efeito mais central relacionado ao uso do paracetamol, quando comparado ao efeito periférico, uma vez que essas enzimas possuem grande expressão no Sistema Nervoso Central. Com isso, tem-se como resultado as propriedades antitérmicas e analgésicas do medicamento (TORRES, et al., 2019).

De acordo com Wannmacher (2005, p. 02), o paracetamol é considerado seguro em doses terapêuticas. A hepatotoxicidade relacionada aos metabólitos ativos e outros fatores apresenta considerável variação individual e associa--se à 
superdosagem absoluta (foi vista com dose única de io a is gramas) acidental ou deliberada. A suscetibilidade à hepatotoxicidade fica aumentada por consumo de álcool, idade, etnia e interações medicamentosas com outros fármacos lesivos ao fígado, mas, mesmo na presença desses fatores, é rara com doses terapêuticas. $O$ amplo uso dos medicamentos sem orientação, juntamente ao desconhecimento dos malefícios e a utilização não racional dos mesmos, é uma das principais causas responsáveis pelas intoxicações humanas registradas no país. A Organização Mundial de Saúde (2002, p.I)

O papel paliativo ou curativo de um medicamento não se limita somente à acessibilidade, deve ser acompanhado de informações apropriadas, sejam verbais ou por escritas, com intervenção de forma decisiva na sua utilização no intuito de minimizar os riscos previsíveis. Dentro deste contexto, pode ser dito que um medicamento é a soma do produto farmacêutico com a informação sobre o mesmo (GOMES, 2003 aput BOLZAN, 2008).

Desta forma associa-se a Atenção Farmacêutica como umas das principais ações voltadas para orientação do uso racional de medicamentos, responsável pela educação da população; prática profissional do farmacêutico em que o paciente é o principal beneficiário (OMS, 2002, p.I).

\section{OBJETIVOS}

\section{I.I. Objetivo Geral}

Investigar a atuação do profissional farmacêutico frente ao uso de paracetamol como medicamento de venda livre.

\section{I.2. Objetivos Específicos}

- Acompanhar e divulgar a Legislação pertinente a ação do farmacêutico;

- Considerar os riscos de utilização inadequada;

- Orientar sobre o papel do farmacêutico. 


\section{METODOLOGIA}

Este estudo é uma revisão da literatura especializada, realizada através de consultas a livros, periódicos e artigos científicos indexados nos bancos de dados do Scielo, Pubmed e Medline, no período de 2008 à 202I.

\section{JUSTIFICATIVA}

O paracetamol é considerado seguro e eficaz em suas doses terapêuticas, mas quando consumido em doses superiores às recomendadas pode ocasionar a hepatotoxicidade.

\section{REVISÃO DA LITERATURA 4.I. História do Paracetamol}

O produto foi pela primeira vez comercializado em 1955 pelos Laboratórios McNeil, pelas ordens do farmacêutico Robert $\mathrm{McNeil}^{3}$, como um aliviador de dores e febre para crianças, sob o nome registrado de "Tylenol, Elixir das Crianças". «Um Festival de Analgésicos». Chemical Heritage Foundation. Em 1956, os comprimidos de $500 \mathrm{mg}$ de paracetamol foram também colocados à venda no Reino Unido com o nome comercial "Panadol", produzido por Frederick Stearns \& Co, uma filial de Sterling Drug Inc. No início, o Panadol, indicado para o alívio das dores e febre, sem causar irritação gástrica como o ácido acetilsalicílico, só podia ser dispensado aos doentes, mediante a apresentação de uma receita médica. Em junho de 1958 iniciou-se a comercialização de uma nova forma de apresentação (xarope), o "Elixir Panadol", destinado às crianças. Em I963, o paracetamol foi adicionado à Farmacopeia Britânica e desde então o seu uso vulgarizou-se como um agente analgésico com poucos efeitos secundários e com poucas interações medicamentosas.

A patente sobre o paracetamol expirou nos Estados Unidos da América e desde então diversos genéricos ficaram disponíveis no mercado, de acordo com a Ata de Competitividade de Preços e a Lei da Restauração de Patentes, conhecida por

\footnotetext{
${ }^{3}$ Robert Lincoln McNeil, Jr. foi um químico e farmacêutico americano. McNeil foi responsável pela criação do nome do medicamento Tylenol (paracetamol) e sua introdução no mercado farmacêutico no ano de 1955 .
} 
"Hatch-Waxman Act" de 1984, ainda que algumas formas farmacêuticas do Tylenol ficassem protegidas até 2007. Nos Estados Unidos, a patente número 6.126.967 de 3 de setembro de 1988, terminou para todos os tipos de formas de apresentação da substância designada por acetaminofeno. (Wikipédia Enciclopédia).

O paracetamol foi descoberto na Alemanha em $1877^{4}$ é o medicamento mais utilizado para o alívio da dor e da febre na Europa e nos Estados Unidos ${ }^{5}$ consta na Lista de Medicamentos Essenciais da Organização Mundial de Saúde, considerados os mais eficazes e seguros medicamentos para responder às necessidades dum sistema de saúde ${ }^{6}$. O paracetamol encontra-se disponível como medicamento genérico ou sob diversas marcas comerciais. (Hamilton, Richard J. 2013).

Atualmente o paracetamol lidera a lista dos mais encontrados em residências domiciliares. Alguns fatores que motivam a prática da automedicação são: a insatisfação com a demora e a baixa qualidade do atendimento dos serviços de saúde, experiências anteriores com o medicamento, indicações de amigos e familiares (NAVES et al., 2010; LUCAS et al., 2014).

\subsection{Caracterização do Paracetamol (Acetaminofeno)}

O mecanismo de ação analgésica não está totalmente determinado, o paracetamol pode atuar inibindo a síntese de prostaglandinas ao nível do Sistema Nervoso Central e em menor grau bloqueando a geração do impulso doloroso ao nível periférico, sendo esta decorrente também da inibição da síntese de prostaglandinas ou da inibição da síntese ou da ação de outras substâncias que sensibilizam os nociceptores antiestímulos mecânicos ou químicos. A ação antipirética é devido ao paracetamol atuar ao nível central sobre o centro hipotalâmico regulador da temperatura para produzir uma vasodilatação periférica que dá lugar a um aumento do fluxo de sangue na pele, de sudorese e da perda de

\footnotetext{
4 Mangus, Brent C.; Miller, Michael G. (2005). Pharmacology application in athletic training. Philadelphia, Pennsylvania: F.A. Davis. p. 39.

${ }^{5}$ Aghababian, Richard V. (22 de outubro de 2010). Essentials of emergency medicine. [S.1.]: Jones \& Bartlett Publishers. p. 8I4. ISBN 978-1-4496-1846-9.

${ }^{6}$ «WHO Model List of Essential Medicines (19th List)»
} 
calor e ação central provavelmente relacionada com a inibição da síntese de prostaglandinas no hipotálamo.

Embora o paracetamol foi utilizado clinicamente há mais de um século, existe debates sobre o seu principal local de ação, que pode ser a inibição da síntese de prostaglandinas ou a ativação de receptores canabióides (ANDERSON, 2008). O paracetamol é indicado para dor suave à moderada, como as dores de cabeça, resfriados, gripe, dores musculares, entorses, dores nas costas (incluindo dor lombar), dismenorreia, dores de artrite menor e dores de dente. $\mathrm{O}$ tratamento desse fármaco compreende na utilização da cefaleia, mialgia moderada, artralgia, dor crônica do câncer, dor pós-parto e pós-operatório e febre (SILVA, 2006).

\subsubsection{Consumo Indiscriminado e Irracional do Paracetamol}

Os medicamentos de venda livre são conhecidos também como Medicamentos Isentos de Prescrição (MIP) e são os de primeira escolha pela população para enfermidades (PINTO, et al., 2015). Para Reis e Ojeda (2002) hoje em dia há uma equivocada concepção populacional referente aos AINE e aos analgésicos, criou-se a ilusão de que esses medicamentos proporcionam a resolução e cura de qualquer enfermidade sem que haja malefícios advindos desse ato. No entanto, há uma falha nessa concepção, podendo induzir ao uso desnecessário do medicamento. Além de aumentar os riscos dos efeitos adversos, perpetua o hábito de se automedicar e de indicar a droga a outras pessoas.

O Acetoaminofeno, conhecido como Paracetamol, é um analgésico não esteroide (AINE), quando utilizado de forma prolongada pode gerar problemas no fígado e rins, podendo causar insuficiência hepática aguda (IHA) e/ou insuficiência renal. É um medicamento de administração oral que pode ser adquirido em formato de pastilha, comprimido, cápsula ou drágea, bem como em fórmulas líquidas (MEIRA, et al., 2013). A toxicidade induzida pelo paracetamol é uma consequência muito comum da overdose e pode levar à insuficiência hepática aguda (ALF). Outro estudo nos Estados Unidos mostrou dentre os 662 pacientes de cuidados terciários, 48\% apresentaram hepatotoxicidade grave por paracetamol e não tinham intenção de envenenarem-se, tomaram o medicamento para fins terapêuticos; estes pacientes 
tendem a ser mais velhos comparados aos pacientes intencionais (tentativa de suicídio) (LARSON et al., 2005).

Quando comparado ao que tange a Pandemia Covid-ı9, o Paracetamol foi visto como um aliado no tratamento inicial dos sintomas causados pelo vírus - como febre e dor, sendo dessa forma, indicado por diversas organizações de saúde como a medicação de escolha. Vale ressaltar que mesmo diante da segurança oferecida pelo medicamento quando utilizado em dose e periodicidade adequadas, é preciso atenção para possíveis interações com álcool, alimentos e outras drogas. Somado a isso, salienta-se que em caso de manutenção da febre ou piora dos sintomas é preciso recorrer a atendimento médico (REMIÃO, 2020).

Figura :. Causadores de lesões no fígado.

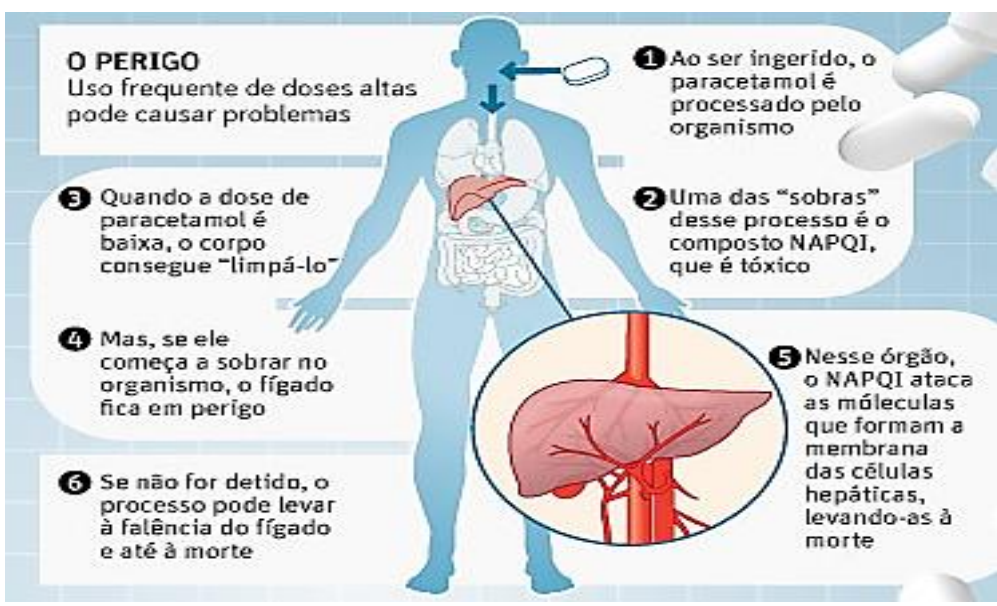

Fonte: http://biblioteca.cofen.gov.br. Acesso 20 de agosto 2021.

\section{I.3. Doses terapêuticas administradas}

A dose terapêutica convencional depende da via de administração e varia entre 325 a $1000 \mathrm{mg}$ em adultos, administrados por períodos de tempo que variam entre 4-6 horas, até um máximo de $4000 \mathrm{mg}$ por dia. Em crianças, a dose única varia entre 40 a $480 \mathrm{mg}$ até um máximo de 5 doses diárias, dependendo do peso e da idade (Goldman e Gilman, 200I). A dose entre 5-15 mg/ $\mathrm{kg}$ por via oral ou de $15^{-20} \mathrm{mg} / \mathrm{kg}$ por via retal num máximo de $75 \mathrm{mg} / \mathrm{kg} /$ dia é considerada como a dose máxima eficaz e segura.

Uma dose de $90 \mathrm{mg} / \mathrm{kg} /$ dia é considerada por muitas autoridades como dosagem “supraterapêutica” (MARZUILLO et al., 2014). É recomendado aumentar o intervalo 
de dosagem para 6 horas em doentes com insuficiência renal moderada (TFG entre Io-50 $\mathrm{ml} / \mathrm{min}$ ), e de 8 em 8 horas em doentes com insuficiência renal grave (TFG).

Figura 3. Intoxicação por Paracetamol.

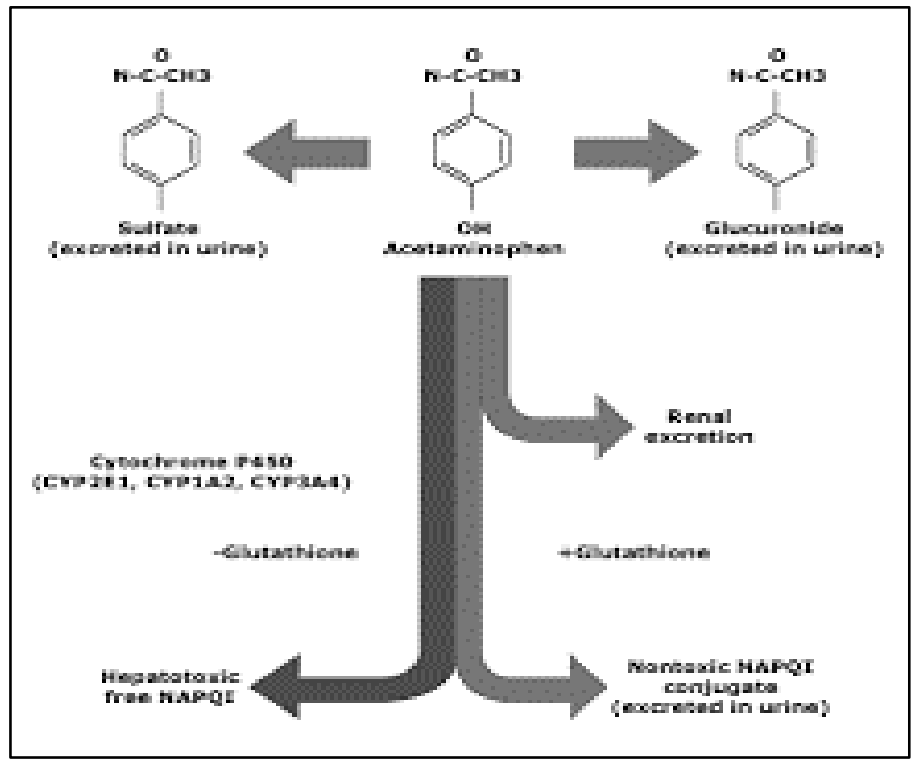

Fonte: https://www.google.com

Segundo a American Academy of Pediatrics (APP) descreve a intoxicação do paracetamol em quatro fases. A primeira consiste na anorexia, náuseas, vômitos, mal-estar, sudorese e o que pode provocar a administração de doses adicionais do paracetamol. Na segunda fase, os sinais da primeira são substituídos pela dor no quadrante superior direito ou sensibilidade, aumento do fígado e oligúria em alguns pacientes; a bilirrubina e os níveis de enzimas hepáticas tornam elevados e o tempo de protrombina prolongado.

$\mathrm{Na}$ terceira fase, geralmente 3 a 5 dias no curso, anorexia, náuseas, vômitos e mal-estar reaparecem junto com sinais de insuficiência hepática, incluindo icterícia, hipoglicemia, encefalopatia, coagulopatia, insuficiência renal e cardiopatia. A quarta fase está associada com a recuperação ou a progressão para a morte por insuficiência hepática completa.

\section{I.4. Legislação e intervenção do profissional farmacêutico no uso do Paracetamol.}

No Brasil a menção aos medicamentos isentos de prescrição (MIP) foi feita pela primeira vez na Lei $\mathrm{n}^{-}$5.991, de 17 de dezembro de 1973, que dispõe sobre o 
controle sanitário de medicamentos. Esses medicamentos podiam ser comercializados em estabelecimentos hoteleiros e similares para atendimento exclusivo de seus usuários. Foi somente em 2003 que a ANVISA publicou a RDC no 138, de 29 de maio de 2003 que dispõe sobre o enquadramento na categoria de venda de medicamentos, onde foi apresentado a Lista de Grupos e Indicações Terapêuticas Especificadas (GITE) (Anexo r). O desenvolvimento do GITE baseou-se em critérios como índice terapêutico, toxicidade, legislações internacionais e a lista de medicamentos essenciais (RENAME), todo medicamento que se enquadra dentro do GITE é considerado um MIP (BRASIL, 2003). A RDC no 138, de 29 de maio de 2003 relata também que os medicamentos cujos grupos terapêuticos e indicações terapêuticas não estão expostos no GITE, não são isentos de prescrição médica. Da mesma forma, associações medicamentosas, cujo grupo terapêutico e indicação terapêutica de pelo menos um dos princípios ativos contidos não estiverem presentes no GITE, só poderão ser vendidos sob prescrição médica. Já a venda de medicamentos novos sempre exigirá a prescrição médica. Contudo, a reavaliação do I2 enquadramento na categoria de venda ocorrerá no momento de sua renovação, seguindo os dados da farmacovigilância. Porém, se esses medicamentos já estiverem sendo comercializados nos Estados Unidos da América ou na Europa pelo período mínimo de 5 anos, desde que aprovados pelos seus respectivos órgãos de Vigilância Sanitária, com grupos terapêuticos e indicações terapêutica presentes no GITE, comprovante de enquadramento na categoria de venda isenta de prescrição no país onde o produto é vendido e com apresentação de dados de farmacovigilância poderão requerer o enquadramento na categoria dos medicamento isentos prescrição, a qualquer hora, desde o momento do registro (BRASIL, 2003).

A Resolução do Conselho Federal de Farmácia (CFF) no 357 , de 20 de abril de 200I, aprova o regulamento técnico das Boas Práticas de Farmácia. Essa resolução expõe que o farmacêutico tem responsabilidade sobre a automedicação responsável e para que isso seja realizado, ele deve fomentar informação e educação sanitária ao paciente ou consumidor e desenvolver ações na dispensação e seleção dos MIPs. Em vista disso, devem ser avaliadas as necessidades do paciente por meio de análise dos 
sintomas e das condições individuais com o objetivo de escolher o medicamento e o aconselhamento adequado para cada indivíduo (BRASIL, 20oI).

A Organização Pan-Americana da Saúde (OPAS) define a Atenção Farmacêutica como:

[...] modelo de prática farmacêutica, desenvolvida no contexto da Assistência Farmacêutica. Compreende atitudes, valores éticos, comportamentos, habilidades, compromissos e corresponsabilidades na prevenção de doenças, promoção e recuperação da saúde, de forma integrada à equipe de saúde. É a interação direta do farmacêutico com o usuário, visando uma farmacoterapia racional e a obtenção de resultados definidos e mensuráveis, voltados para a melhoria da qualidade de vida. Esta interação também deve envolver as concepções dos seus sujeitos, respeitadas as suas especificidades biopsicossociais, sob a óptica da integralidade das ações de saúde (OPAS, 2002).

\section{CONCLUSÃO}

Este estudo proporcionou uma ampla visão de que a automedicação pode mascarar e ocultar possíveis diagnósticos além de dificultar e intervir em tratamentos já estabelecidos.

Tomar de uma só vez grandes quantidades de paracetamol pode lhe causar grandes lesões no fígado, algumas irreversíveis, necessitando de transplante. Em outros casos, tomar deliberadamente paracetamol pode resultar em uma hepatite fulminante, com conseqüências fatais.

O farmacêutico tem a responsabilidade de orientar e educar a sociedade, quanto à dose, frequência, duração do tratamento e vias de administração, salientando as possíveis reações adversas e contraindicações. As ações de atenção farmacêutica bem estruturadas constituem uma abordagem imprescindível para a promoção da saúde, através da relação paciente-farmacêutico em busca da otimização dos resultados terapêuticos

\section{REFERÊNCIAS BIBLIOGRÁFICAS}

AMERICAN ACADEMY OF PEDIATRICS: Acetaminophen Toxicity in Children Committee on Drugs Pediatrics, v. I08, n. 4, oct., 2001. Disponível em: $<$ http://pediatrics.aappublications.org/content/ro8/4/iozo.full 〉. Acesso is de agosto 2021. 
ANDERSON, B. Paracetamol (Acetaminophen): mechanisms of action., 2008 oct., 18

(10):915-21. Disponível em: 〈http://www.ncbi.nlm.nih.gov/pubmed/I88I1827〉. Acesso 27 de julho de 202I

ARAUJO A, et al. Paracetamol uma visão farmacológica e toxicológica. Artigo apresentado no $\mathrm{V}$ Seminário de pesquisas e Trabalho de conclusão de curso da Faculdade União de Goyazes. Trindade, 2013.

ANVISA, Agencia Nacional de Vigilância Sanitária https://agenciabrasil.ebc.com.br/saude/noticia/2021-05/anvisa-alerta-para-riscos-douso-indiscriminado-de-paracetamol. Acesso em os de setembro de 2021.

BRASIL, Resolução do CFF no 357 , de 20 de abril de 2001 - Aprova o regulamento técnico das Boas Práticas de Farmácia. Disponível em: 108 Acesso em: io de agosto 202I.

BERTOLINI, A., et al. (2006). Paracetamol: New Vistas of an Old Drug. Blackwell Publishing Inc., 12, pp. 250-275.

GOLDMAN, L. E GILMAN, A. (200I). The pharmacological basis of therapeutics, Rio de Janeiro, McGraw-Hill.

LARSON, A et al. Acetominophen-induced acute liver failure: results of a United States multicenter, prospective study. Hepatology, 2005 dec, 42 (6): 1364-74. Disponível em: 〈http://www.ncbi.nlm.nih.gov/pubmed/r6317692〉. Acesso i9 de setembro de 2021.

MEIRA C, et al. Paracetamol: para além da toxicidade hepática. Associação Cuidados Intermédios Médicos, 2013; 2: 26-31.

MARZUILLO, P., GUARINO, S. E BARBI, E. (2014). Paracetamol: a focus for the general pediatrician. Eur J Pediatr, I73, pp. 415-25.

NAVES, JOS; CASTRO, LLC; CARVALHO, CMS; MERCHÁN-HAMANN, E. Automedicação: uma abordagem qualitativa de suas motivações. Ciência \& saúde coletiva, I5 (Suppl I):1751-1762, jun. 2010.

OPAS - ORGANIZAÇÃO PAN-AMERICANA DA SAÚdE. Consenso Brasileiro de Atenção Farmacêutica: proposta. Brasília, DF, 2002. 24p.

ORGANIZACIÓN MUNDIAL DE LA SALUD. Perspectivas políticas sobre medicamentos de la OMS. Promoción del uso racional de medicamentos: componentes centrales. Geneva: Organización Mundial de la Salud; 2002. 
Organização Pan--Americana da Saúde/Organização Mundial da Saúde - Brasil, 2010. Disponível em: I4 de setembro de 202I.

PINTO NQO, et al. Atuação do profissional farmacêutico frente ao uso Indiscriminado de Paracetamol como medicamento de venda indiscriminado de Paracetamol como medicamento de venda

livre.FACIDER Revista Cientifica. 2015; 7: 1-20

REMIÃO F. O paracetamol e a COVID-I9. Revista de Ciência Elementar. 2020; v8(02): 023.

SILVA, Penildon. Farmacologia. 7.ed. Rio de Janeiro: Guanabara Koogan S.A., 2006, p.44I- 453 .

TORRES VL, et al. Hepatotoxicidade do Paracetamol e fatores predisponentes. Revista de ciências da saúde Nova Esperança. 2019; 17(I): 93-99

WANMMACHER, L. Medicamentos de Uso Corrente no Manejo de Dor e Febre Uso Racional de Medicamentos: Temas Selecionados, v. o8, 2010.

https://pt.wikipedia.org/wiki/Paracetamol Acesso em 20 de agosto de 202I.

〈http://www.ncbi.nlm.nih.gov/pubmed/16317692〉

http://www.cff.org.br/cebrim/arquivo/7360/201203161748131,pdt. 